\title{
Robust Adaptive PI Control of Wave Energy Converters with Uncertain and Nonlinear Dynamics
}

This paper was downloaded from TechRxiv (https://www.techrxiv.org).

\section{LICENSE}

CC BY 4.0

SUBMISSION DATE / POSTED DATE

$10-01-2022$ / 13-01-2022

\section{CITATION}

Hoai Nam, Nguyen (2022): Robust Adaptive PI Control of Wave Energy Converters with Uncertain and Nonlinear Dynamics. TechRxiv. Preprint. https://doi.org/10.36227/techrxiv.18096101.v1

$\mathrm{DOI}$

10.36227/techrxiv.18096101.v1 


\title{
Robust Adaptive PI Control of Wave Energy Converters with Uncertain and Nonlinear Dynamics
}

\author{
Hoai-Nam Nguyen $^{\text {a }}$ \\ a SAMOVAR - Télécom SudParis - IP Paris, 19 rue Marguerite Perey. 91120 \\ Palaiseau, France
}

\begin{abstract}
There exist several approaches to design the optimal control strategy to harvest wave energy with a point absorber. However they are generally based on the assumption that the WEC and the PTO dynamics are well-known. In the practical WEC control implementation, this is generally not the case. The objective of this paper is to design a robust optimal control strategy that can take into account the uncertain WEC and PTO dynamics. Our choice is a robust adaptive PI control law. The proposed controller is validated and compared through simulation for irregular sea states.
\end{abstract}

Key words: Wave Energy Converter (WEC), Robust Adaptive PI, Uncertain Dynamics, Semidefinite Programming (SDP)

\section{Introduction}

The ocean is an almost inexhaustible source of energy and represents one of the most reliable and promising forms of renewable energy [4], [5]. According to [18], the ocean holds approximately $8000-80000 \mathrm{TWh} /$ year or $1-10 \mathrm{TW}$. Comparing this to the world's annual energy consumption of approximately 148000 TWh in 2008, it is shown [9] that wave energy could play an important role in the world's energy portfolio. Currently, several wave energy converter (WEC) concepts are under investigation, but wave energy is still far from being competitive with other forms of renewable energy such as wind or solar. The production cost per unit of power for WEC systems is hampered by two fundamental problems:

Email address: hoai-nam.nguyen@telecom-sudparis.eu (Hoai-Nam Nguyen). 
(1) High cost of maintenance. Under extreme conditions, WEC systems experience large amplitude motions, wave over-topping, wave slamming, etc. These physical phenomena increases the risk of device damage.

(2) Low energy conversion rate. This concerns virtually all the WEC concepts, regardless of the width of their absorption band. For a broad category of WECs, however, wave-to-grid efficiency strongly depends on the way the machine, and its Power Take-Off (PTO) system in particular, is controlled.

In contrast with the wind energy sector, there is no industrial standard device for wave energy conversion and this diversity is directly related to the PTO system. Many different types of PTO systems have been studied, and the type of PTO system used in a wave energy converter is often correlated with its type [25]. WEC systems are located in very harsh environment, leading to a high wear-rate and are difficult to access due to their location and/or unfavorably weather conditions. As for the rest of the device, the PTO system should be robust, reliable and should require as little maintenance as possible.

When there is a flexible PTO available, capable of both harvesting and drawing power from the grid, respectively in generator and motor modes, an intermittent switching $P I$-controller [28] can be considered as the standard solution. The $P$ component converts the wave energy into useful energy, while the $I$ component modifies the WEC system natural frequency which allows the absorber to be more often in phase with the incoming waves, at the expense of some power being drawn from the grid. Under the assumptions that the PTO system has no dynamics, and that the WEC dynamics are well-known, mathematical tools involved in the computation of the optimal PI gains are remarkably simplified. There exists an elegant analytical expression for the PI gains as a function of wave frequency in the case of regular waves [5]. Unfortunately, a PTO system without dynamics is unrealistic. In addition, due to friction, viscous and many other forces, the WEC and PTO dynamics are often not well-known. In these cases, to the best of the authors knowledge, there is no available numerical method in the literature to calculate the optimal PI gains. The main objective of this paper is to fill this gap.

It is evident that the PI gains should be adapted in real-time as the sea conditions changes continuously. Adaptive PI control has been already studied in [10], [22], [23]. In most literature, only intermittent adaptive PI control laws are considered, where the control gains are constant within a time window of several minutes, e.g., 30 minutes in [10]. Clearly, such intermittent adaptive control laws are sub-optimal in terms of energy recovery. In [22], [23], a new adaptive PI control algorithm is proposed, for which the PI gains are continuously adapted on-line, on a wave-to-wave basis. The most notable feature of the control law in [22], [23] is that the PTO nonlinear efficiency coefficient is taken into account in the controller design phase. However, it is still assumed 
that the WEC dynamics are well-known and that the PTO system has no dynamics.

In this paper, we continue the research line of [22], [23]. Using a real-time sinusoidal approximation of the wave excitation force, the PI gains are adapted on-line. The uncertain WEC and PTO dynamics are taken into account in the controller design step. The worst case scenario is considered, i.e., the worstcase harvested average power is maximized. In other words, a min-max optimization problem is solved. We then show how to convert this optimization problem into a convex semidefinite programming (SDP) problem, for which there exists several free available software packages [15], [8].

It is worth stressing that even though the mismatch between model and the actual system is inevitable, the issue of robustness in the WEC field is still rarely to be discussed. Among some attempts in the topic of synthesizing robust WEC controllers, a hierarchical control strategy was considered in [6], [31]. The basic idea is to generate a high-level suboptimal velocity reference, and then a low-level servo control loop. To generate the velocity profile, only a very particular type of model uncertainties can be considered. In addition, the PTO dynamics were not taken into account.

This paper presents a rigorous treatment of the preliminary results in [19]. In this paper:

(1) Both system and actuator dynamics are uncertain. As a consequence, the nonlinear Morrison force can be addressed. In [19], only the uncertain actuator dynamics case is considered. The system dynamics are assumed to be linear and well-known, see equation (1) in [19].

(2) A procedure to identify an uncertain transfer function is provided. In [19], there is no such procedure.

(3) A proof of Theorem 1 is given. In [19], this theorem is presented without proof.

(4) Theorem 2 and its proof are new.

The paper is organized as follows. Section 2 describes the problem formulation and some preliminaries concerning the SDP optimization problem. A procedure to calculate the real and imaginary parts of the robust optimal control block under regular waves in the presence of a uncertain WEC and PTO dynamics is presented in Section 3. Section 4 explains how the results of Section 3 can be extended to deal with realistic polychromatic sea states. In Section 5 , the simulation setup is described used to validate the algorithm, and the results are reported. Finally, some conclusions are drawn in Section 6. 


\section{Problem Formulation and Preliminaries}

\subsection{Model of the WEC}

We consider point-absorber WECs consisting of a heaving body that moves in one or two degrees of freedom with respect to a fixed anchor or a submerged body. From the relative motion, useful energy can be extracted. In general, the WEC is axis-symmetric so that the energy conversion rate is almost the same for waves coming from all directions, see Fig. 1.

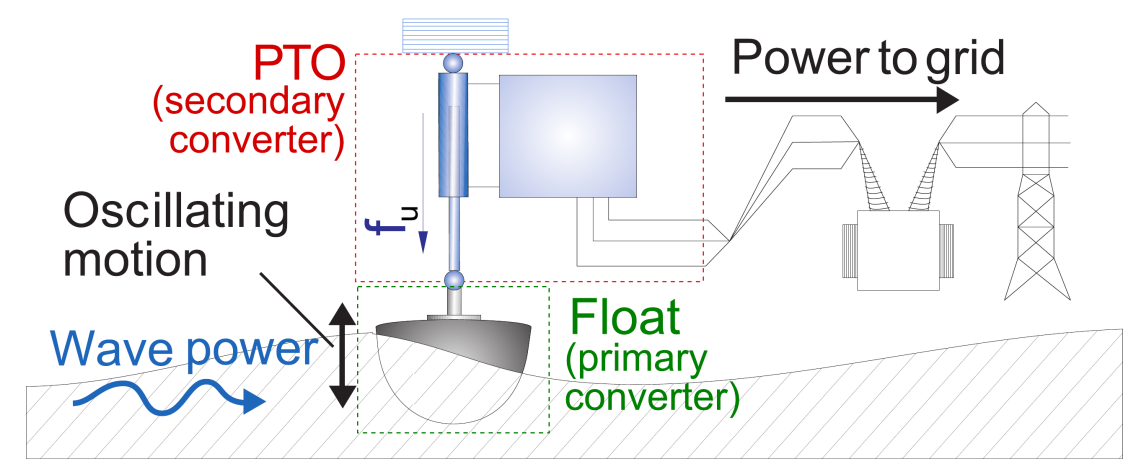

Fig. 1. One-degree-of-freedom floating system for wave energy converter.

The equation of motion for the WEC system can be obtained as,

$$
M_{s} \dot{v}(t)=f_{e x}(t)-f_{p t o}(t)+f_{r}(t)+f_{h d}(t)+f_{v}(t)
$$

where $M_{s}$ is the WEC mass, $v(t)$ is the heaving velocity, $f_{e x}(t)$ is the wave excitation force, and $f_{p t o}(t)$ is the force from the PTO system. The other force terms and their mathematical representation are explained in the following.

$f_{r}(t)$ : Radiation force.

The radiation force due to radiated waves is given as

$$
f_{r}(t)=-M_{\infty} \dot{v}(t)-f_{r h}(t)
$$

where $M_{\infty}$ is the added mass at infinitely high frequency, and

$$
f_{r h}(t)=\int_{0}^{t} h(t-\tau) v(\tau) d \tau
$$

$h(t)$ is the impulse response function of the radiation force, which can be obtained by boundary element methods [3], [13]. Equation (3) can be considered as a linear system, where the input is $v(t)$ and the output is $f_{r h}(t)$. It can be rewritten as,

$$
F_{r h}(j w)=W_{r}(j w) V(j w)
$$


where $F_{r h}(j w), W_{r}(j w), V(j w)$ are the Fourier transform of $f_{r h}(t), h(t)$ and $v(t)$, respectively.

$f_{h d}(t)$ : Hydrostatic force.

The hydrostatic force gives stiffness force as deviation from hydrostatic equilibrium. This force is obtained as

$$
f_{h d}(t)=-K_{h d} p(t)
$$

where $K_{h d}$ is the hydrostatic coefficient, $p(t)=\int_{0}^{t} v(\tau) d \tau$ is the buoy position. $f_{v}(t)$ : Viscous drag force.

The force due to viscous forces is given as a quadratic function of buoy velocity

$$
f_{v}(t)=K_{v}|v(t)| v(t)
$$

where $K_{v}$ is the Morison coefficient. Note that this is a simplification of the quadratic term of the Morison equation, which usually considers the relative velocity of buoy and water.

There are other forces such as friction, mooring forces. However they are not considered in the paper. The motion response of the WEC system is given by the following model

$$
M \dot{v}(t)+\int_{0}^{t} h(t-\tau) v(\tau) d \tau+K_{h d} \int_{0}^{t} v(\tau) d \tau+K_{v}|v(t)| v(t)=f_{\text {ex }}(t)-f_{p t o}(t)
$$

with $M=M_{s}+M_{\infty}$.

For convenience of control design, the WEC model is typically expressed in the frequency domain as

$$
V(j w)=\frac{1}{Z_{i}(j w)}\left(F_{e x}(j w)-F_{p t o}(j w)\right)
$$

where $Z_{i}(j w)$ is the intrinsic impedance of the system. $F_{e x}(j w)$ and $F_{p t o}(j w)$ represent, respectively, the Fourier transform of $f_{e x}(t)$, and of $f_{p t o}(t)$. Note that $\left.Z_{i}(j w)\right)$ is uncertain due to the un-modeled forces, due to the presence of the viscous drag force, as well as the variation of the WEC parameters such as $K_{h d}$.

Define $F_{u}(j w)$ as the control input. The PTO force $F_{p t o}(j w)$ is related to $F_{u}(j w)$ as, see Fig. 1

$$
F_{p t o}(j w)=W_{p t o}(j w) F_{u}(j w)
$$

where $W_{p t o}(j w)$ is the PTO transfer function. $W_{p t o}(j w)$ is also uncertain in this study. 


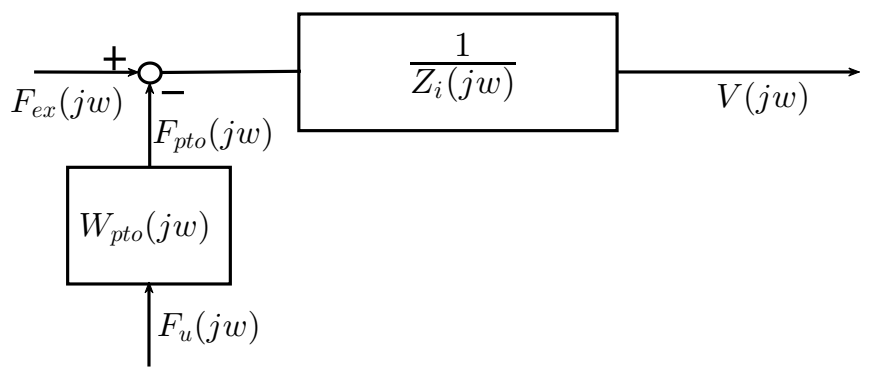

Fig. 2. Structure of the considered mathematical WEC model.

Combining (8), (9), one gets

$$
\left\{\begin{array}{l}
V(j w)=\frac{1}{Z_{i}(j w)}\left(F_{e x}(j w)-W_{p t o}(j w) F_{u}(j w)\right) \\
F_{p t o}=W_{p t o}(j w) F_{u}(j w)
\end{array}\right.
$$

Equation (10) will serve as a basic for the results in the next sections.

\subsection{Control Objective}

The objective for the WEC system is to select the input signal $f_{u}(t)$ that maximizes the harvested average power $P_{a}$

$$
P_{a}=\frac{1}{T} \int_{0}^{T} P(t) d t
$$

where $P(t)=f_{\text {pto }}(t) v(t)$ is the instantaneous power. In the literature, it is generally assumed that $W_{p t o}(j w)=1, \forall w$, and that the WEC dynamics are well known. With these assumptions, the mathematical tool involved to calculate the optimal control signal $f_{u}(t)$ is greatly simplified, see e.g., [5]. As written in Introduction, many different types of PTO systems exist, and the type of PTO chosen for a particular WEC is often strongly related with the type of converter. For the point absorber type of converter in the paper, a linear electrical generator or a hydraulic system are generally used [25]. Unfortunately, the dynamics of these PTO systems are relatively long with respect to the WEC dynamics, and therefore can not be neglected. In addition, due to viscous, frictions and several other forces, the WEC and PTO dynamics are 
not completely known. Therefore, it is important in optimal control of WEC systems to take these uncertainties into account.

Define $R_{i}(w), X_{i}(w)$ and $R_{p t o}(w), X_{p t o}(w)$, respectively, as the real and the imaginary parts of $Z_{i}(j w)$ and of $W_{p t o}(j w)$, i.e.

$$
\left\{\begin{array}{l}
Z_{i}(j w)=R_{i}(w)+j X_{i}(w), \\
W_{p t o}(j w)=R_{p t o}(w)+j X_{p t o}(w)
\end{array}\right.
$$

Note that $R_{i}(w), X_{i}(w)$ and $R_{p t o}(w), X_{p t o}(w)$ are not well-known due to the uncertainties of $Z_{i}(j w), W_{p t o}(j w)$. Details of how to obtain a set of possible values of $R_{i}(w), X_{i}(w)$ and $R_{p t o}(w), X_{p t o}(w)$ are described in Section 2.4.

In summary, the control objective for the WEC system is to calculate the control input $f_{u}(t)$ that maximizes the worst-case harvested average power $P_{a}$ in the presence of the uncertain WEC and PTO systems (12). In other words, we aim to solve the following max-min problem

$$
\max _{f_{u}} \min _{R_{i}, X_{i}, R_{p t o}, X_{p t o}}\left\{P_{a}\right\}
$$

Remark: Fuzzy control [16], [17] might be an alternative solution for the case of uncertain WEC and PTO systems. However, this approach requires an online estimation of the model.

\subsection{Notation and Preliminaries}

Some standard notations are introduced in this section. For symmetric matrices, the symbol $(*)$ denotes each of its symmetric blocks. We denote $\mathbb{R}^{n \times m}$ the set of real $n \times m$ matrices. For any real symmetric matrix $X$, the notation $X \succeq 0$ indicates that all eigenvalues of $X$ are nonnegative.

In the following, we recall some basic definitions of semidefinite programming (SDP) that we will use later to design a robust optimal control law.

A linear matrix inequality is a condition of the type

$$
F(x) \succeq 0
$$

where $x \in \mathbb{R}^{n}$ is a vector variable, and the matrix $F(x)$ is affine in $x$, that is

$$
F(x)=F_{0}+\sum_{i=1}^{n} F_{i} x_{i}
$$


with symmetric matrices $F_{i} \in \mathbb{R}^{m \times m}$.

LMIs can either be understood as feasibility conditions or constraints for optimization problems. Optimization of a linear function over LMI constraints is called SDP, which is an extension of linear programming [2], [21]. Nowadays, a main benefit in using LMIs is that for solving an LMI problem, several polynomial time algorithms were developed and implemented in free available software packages, such as YALMIP [15], CVX [8], etc.

The Schur complement is a very useful tool for matrix inequalities. It states that the following nonlinear condition

$$
\left\{\begin{array}{l}
U(x) \succeq 0, \\
P(x)-Q(x)^{T} U(x)^{-1} Q(x) \succeq 0
\end{array}\right.
$$

can be equivalently written in the LMI form

$$
\left[\begin{array}{ll}
P(x) & Q(x)^{T} \\
Q(x) & U(x)
\end{array}\right] \succeq 0
$$

Hence the Schur complement allows to converts certain nonlinear matrix inequalities into a higher dimensional LMI condition.

\subsection{Identification of Uncertain Transfer Functions}

In this section, a transfer function and/or an intrinsic impedance identification problem is considered. Namely, for a given uncertain system $W_{s}(j w)$, we are looking for a set of all possible values of the real part and of the imaginary part of the transfer function, representing the plant under all relevant operating conditions, on which to base the robust optimal design. It is clear that the value set might be non-convex due to nonlinearies, parameter variations, noise and disturbances, etc.

Denote $U(j w), Y(j w)$, respectively, as the input and output of the considered system, i.e.,

$$
Y(j w)=W_{s}(j w) U(j w)
$$

A straightforward way to obtain the value set is to simulate the system (15) with an input $u(t)=A \sin (w t)$ for several different amplitudes $A$, and then to measure the amplitude $B$ and the phase $\delta$ of the output $y(t)=B \sin (w t+\delta)$. However direct measurements of $B$ and $\delta$ are difficult due to nonlinearies, noises, uncertainties [14], [29]. 
It is worth noticing that the problem of identifying the value set of uncertain systems is well-known [7], [14], [29]. There exist several methods in the literature such as the Lissajou figure method [7], Fourier integral method [14], or correlation method [29]. Due to its simplicity, the Fourier integral approach is chosen in this study. This method is summarized in the following in order to make the paper clearer and more self-contained.

It is assumed that the output measurement $y(t)$ is given as

$$
y(t)=B \sin (w t+\delta)+e(t)
$$

where $e(t)$ is the measurement noise. $e(t)$ is uncorrelated with $u(t)$. Define two new signals

$$
y_{s}=\frac{1}{\mathbf{n} T} \int_{0}^{\mathbf{n} T} y(t) \sin (w t) d t, y_{c}=\frac{1}{\mathbf{n} T} \int_{0}^{\mathbf{n} T} y(t) \cos (w t) d t
$$

where $T=\frac{2 \pi}{w}$ is the period and $\mathbf{n}$ is any large positive integer. One has

$$
\begin{aligned}
y_{s} & =\frac{1}{\mathbf{n} T} \int_{0}^{\mathbf{n} T} y(t) \sin (w t) d t \\
& =\frac{1}{\mathbf{n} T} \int_{0}^{\mathbf{n} T} B \sin (w t+\delta) \sin (w t) d t+\frac{1}{\mathbf{n} T} \int_{0}^{\mathbf{n} T} e(t) \sin (w t) d t
\end{aligned}
$$

Because $e(t)$ is uncorrelated with $u(t)$, one gets $\frac{1}{\mathbf{n} T} \int_{0}^{\mathbf{n} T} e(t) \sin (w t) d t \approx 0$. Hence

$$
y_{s} \approx \frac{B}{2} \cos (\delta)-\frac{B}{2 \mathbf{n} T} \int_{0}^{\mathbf{n} T} \cos (2 w t+\delta) d t \approx \frac{B}{2} \cos (\delta)
$$

Analogously, $y_{c} \approx \frac{B}{2} \sin (\delta)$. Therefore

$$
B=2 \sqrt{y_{c}^{2}+y_{s}^{2}}, \delta=\arctan \left(\frac{y_{c}}{y_{s}}\right)
$$

Once the amplitude and the phase of the output $y(t)$ are obtained, the frequency response of $W_{s}(j w)$ is computed as

$$
\left\{\begin{array}{l}
\left|W_{s}(j w)\right|=\frac{B}{A}, \angle W_{s}(j w)=\delta, \\
R_{s}(w)=\left|W_{s}(j w)\right| \cos (\delta), X_{s}(w)=\left|W_{s}(j w)\right| \sin (\delta)
\end{array}\right.
$$

where $\left|W_{s}(j w)\right|, \angle W_{s}(j w), R_{s}(w), X_{s}(w)$ are, respectively, the amplitude, the phase, the real part, and the imaginary part of $W_{s}(j w)$.

In summary, for uncertain system $W_{s}(j w)$, the value set of the real part $R_{s}$ and of the imaginary part $X_{s}$ can be obtained using Algorithm 1. 
Algorithm 1: Calculation of the value set of the part $R_{s}$ and the imaginary part $X_{s}$ of the uncertain transfer function $W_{s}$ (or of the intrinsic impedance)

1: Select a reasonable finite but large set of frequency samples $0<w_{1}<$ $\ldots<w_{N}$.

2: Select a reasonable finite but large set of amplitude of the input $0<A_{1}<$ $A_{2}<\ldots<A_{L}$.

3: For each frequency $w_{l}, \forall l=\overline{1, N}$, and each amplitude $A_{k}, \forall k=\overline{1, L}$ in the chosen set

4: Compute the amplitude $B$ and the phase $\delta$ of the output as in (17).

5: $\quad$ Compute $R_{s}^{(l, k)}, X_{s}^{(l, k)}$ as in (18).

6: The value sets for $R_{s}$, and $X_{s}$ are obtained as the union of $R_{s}^{(l, k)}$, and $X_{s}^{(l, k)}, \forall l=\overline{1, N}, \forall k=\overline{1, L}$ respectively.

Using Algorithm 1, Fig. 3 presents the value sets of the real and imaginary parts of the WEC intrinsic impedance $Z_{i}(j w)$. A zoom of the value set of $R_{i}(w)$ and $X_{i}(w)$ for one particular frequency $w=6(\mathrm{rad} / \mathrm{s})$ is also presented. The considered frequency interval is $1 \leq w \leq 10$, and the amplitude $1 \leq A \leq 15$. The parameters of the WEC system are given in Section 5.

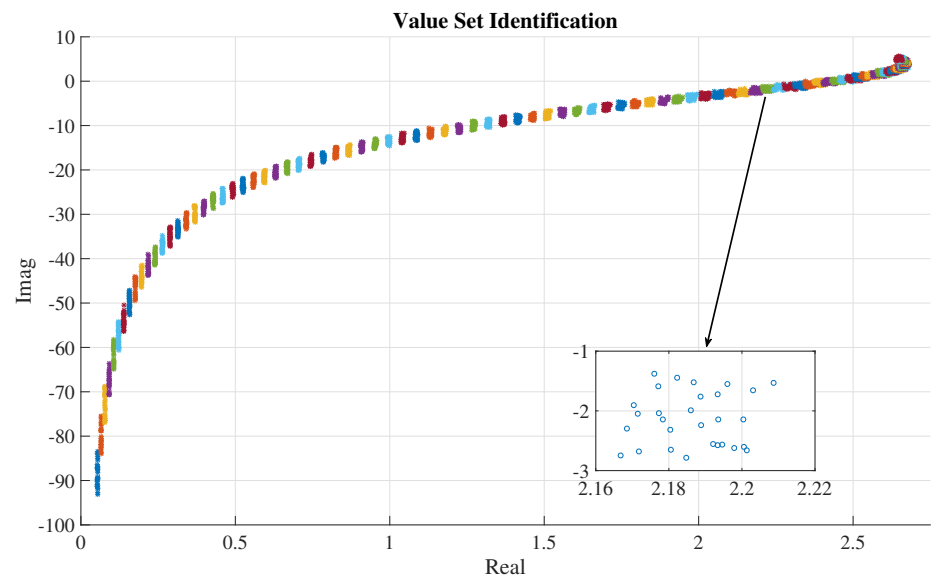

Fig. 3. Value set of the real part $R_{i}(w)$, and of the imaginary part $X_{i}(w)$ of the WEC intrinsic impedance $Z_{i}(j w)$.

\section{Robust Optimal Control in Regular Waves}

\subsection{Average power}

For regular waves, the wave excitation force is given as

$$
f_{e x}(t)=A \sin (w t+\phi)
$$


where $A, w, \phi$ are the amplitude, the angular wave frequency, and the phase angle, respectively. Without loss of generality, $\phi$ can be set to be zero.

In this work, the control input is assumed to be a linear function of the velocity, i.e.,

$$
F_{u}(j w)=Z_{c}(j w) V(j w)
$$

where $Z_{c}(j w)$ can be seen as a load impedance, and designed following impedance matching principles [5].

Define $R_{c}(w), X_{c}(w)$ as the real and the imaginary parts of $Z_{c}(j w)$, i.e.,

$$
Z_{c}(j w)=R_{c}(w)+j X_{c}(w)
$$

For simplicity, the argument $w$ will be omitted from $R_{i}, X_{i}, R_{p t o}, X_{p t o}, R_{c}, X_{c}$, whenever possible.

The following theorem holds.

Theorem 1: Given the wave excitation force (19), and the control input (20), the average power is computed as

$$
P_{a}=\frac{A^{2}\left(R_{p t o} R_{c}-X_{p t o} X_{c}\right)}{2\left(\left(R_{i}+R_{p t o} R_{c}-X_{p t o} X_{c}\right)^{2}+\left(X_{i}+R_{p t o} X_{c}+X_{p t o} R_{c}\right)^{2}\right)}
$$

Proof: Using (10), (20), the WEC velocity is calculated as

$$
V(j w)=W(j w) F_{e x}(j w)
$$

where $W(j w)=\frac{1}{Z_{i}(j w)+W_{p t o}(j w) Z_{c}(j w)}$ is the transfer function from $F_{e x}(j w)$ to $V(j w)$.

As system (23) is linear, one obtains

$$
v(t)=A_{w} A \sin \left(w t+\theta_{w}\right)
$$

where $A_{w}=|W(j w)|$ and $\theta_{w}=\angle W(j w)$ are the amplitude and the phase of $W(j w)$, evaluated at frequency $w$, respectively.

Analogously, using (20), one gets

$$
f_{u}(t)=A_{c} A_{w} A \sin \left(w t+\theta_{w}+\theta_{c}\right)
$$

and, using (10)

$$
f_{p t o}(t)=A_{p t o} A_{c} A_{w} A \sin \left(w t+\theta_{w}+\theta_{c}+\theta_{p t o}\right)
$$

where $A_{c}=\left|W_{c}(j w)\right|, \theta_{c}=\angle W_{c}(j w)$, and $A_{p t o}=\left|W_{p t o}(j w)\right|, \theta_{p t o}=\angle W_{p t o}(j w)$ are, respectively, the amplitude and the phase of $W_{c}(j w)$ and of $W_{p t o}(j w)$ at $w$. 
Combining (24), (26), the instantaneous power is computed as

$$
\begin{aligned}
P(t) & =v(t) f_{p t o}(t) \\
& =A_{p t o} A_{c} A_{w}^{2} A^{2} \sin \left(w t+\theta_{w}\right) \sin \left(w t+\theta_{w}+\theta_{c}+\theta_{p t o}\right)
\end{aligned}
$$

and the average power

$$
P_{a}=\frac{1}{T} \int_{0}^{T} P(t) d t
$$

It is clear that only the relative phase shift of two sines is required for the calculation of $P_{a}$. Hence $\theta_{w}$ can be omitted in (27) without loss of generality, i.e.,

$$
P(t)=A_{p t o} A_{c} A_{w}^{2} A^{2} \sin (w t) \sin (w t+\theta)
$$

with

$$
\theta=\theta_{c}+\theta_{\text {pto }}
$$

It is also clear that it suffices to calculate $P_{a}$ for one period $T=\frac{2 \pi}{w}$, since the wave (19) is periodic. Hence

$$
P_{a}=\frac{w}{2 \pi} A_{p t o} A_{c} A_{w}^{2} A^{2} \int_{0}^{\frac{2 \pi}{w}} \sin (w t) \sin (w t+\theta) d t
$$

and thus, by using the following product-to-sum trigonometric identity

$$
\sin (\alpha) \sin (\beta)=\frac{1}{2}(\cos (\alpha-\beta)-\cos (\alpha+\beta))
$$

one gets

$$
\begin{aligned}
P_{a} & =\frac{w}{4 \pi} A_{p t o} A_{c} A_{w}^{2} A^{2} \int_{0}^{\frac{2 \pi}{w}}(\cos (\theta)-\cos (2 w t+\theta)) d t \\
& =\left.\frac{w}{4 \pi} A_{p t o} A_{c} A_{w}^{2} A^{2}\left(\cos (\theta) t-\frac{\sin (2 w t+\theta)}{2 w}\right)\right|_{0} ^{\frac{2 \pi}{w}}
\end{aligned}
$$

or equivalently

$$
P_{a}=\frac{1}{2} A_{p t o} A_{c} A_{w}^{2} A^{2} \cos (\theta)
$$

Using (30), one has

$$
\begin{aligned}
\cos (\theta) & =\cos \left(\theta_{c}+\theta_{p t o}\right) \\
& =\cos \left(\theta_{c}\right) \cos \left(\theta_{p t o}\right)-\sin \left(\theta_{c}\right) \sin \left(\theta_{p t o}\right)
\end{aligned}
$$

and thus, with (31)

$$
P_{a}=\frac{1}{2} A_{p t o} A_{c} A_{w}^{2} A^{2}\left(\cos \left(\theta_{c}\right) \cos \left(\theta_{p t o}\right)-\sin \left(\theta_{c}\right) \sin \left(\theta_{p t o}\right)\right)
$$

The following equations hold

$$
\left\{\begin{array}{l}
R_{c}=A_{c} \cos \left(\theta_{c}\right), X_{c}=A_{c} \sin \left(\theta_{c}\right), \\
R_{p t o}=A_{p t o} \cos \left(\theta_{p t o}\right), X_{p t o}=A_{p t o} \sin \left(\theta_{p t o}\right)
\end{array}\right.
$$


and with

$$
A_{w}^{2}=\frac{1}{\left(R_{i}+R_{p t o} R_{c}-X_{p t o} X_{c}\right)^{2}+\left(X_{i}+R_{p t o} X_{c}+X_{p t o} R_{c}\right)^{2}}
$$

Equation (32) is rewritten as

$$
P_{a}=\frac{A^{2}\left(R_{p t o} R_{c}-X_{p t o} X_{c}\right)}{2\left(\left(R_{i}+R_{p t o} R_{c}-X_{p t o} X_{c}\right)^{2}+\left(X_{i}+R_{p t o} X_{c}+X_{p t o} R_{c}\right)^{2}\right)}
$$

The proof is complete.

Remark: Consider the limiting case when the PTO has no dynamics. This implies that $W_{p t o}(j w)=1, \forall w$ and hence $R_{p t o}=1, X_{p t o}=0$. Equation (22) becomes

$$
P_{a}=\frac{A^{2} R_{c}}{2\left(\left(R_{i}+R_{c}\right)^{2}+\left(X_{i}+X_{c}\right)^{2}\right)}
$$

Equation (33) is obtained in [5]. Hence the result in [5] is a particular case of Theorem 1 with $W_{p t o}(j w)=1$.

\subsection{Optimal Control - Nominal Case}

In this section, we consider the case when the WEC and PTO dynamics are perfectly known.

Using (22), the next step is to calculate $R_{c}, X_{c}$ to maximize the harvested average power $P_{a}$. By taking the partial derivative of $P_{a}$ with respect to $R_{c}, X_{c}$, and then by solving the following system of equations

$$
\left\{\begin{array}{l}
\frac{\partial P_{a}}{\partial R_{c}}=0 \\
\frac{\partial P_{a}}{\partial X_{c}}=0
\end{array}\right.
$$

one obtains

$$
\left\{\begin{array}{l}
R_{c}^{*}=\frac{R_{p t o} R_{i}-X_{p t o} X_{i}}{R_{p t o}^{2}+X_{p t o}^{2}} \\
X_{c}^{*}=-\frac{R_{p t o} X_{i}+X_{p t o} R_{i}}{R_{p t o}^{2}+X_{p t o}^{2}}
\end{array}\right.
$$

with the corresponding average power

$$
P_{a}^{*}=\frac{A^{2}}{8 R_{i}}
$$

and

$$
\left\{\begin{array}{l}
R_{c}^{* *}=-\frac{R_{p t o} R_{i}+X_{p t o} X_{i}}{R_{p t o}^{2}+X_{p t o}^{2}}, \\
X_{c}^{* *}=\frac{-R_{p t o} X_{i}+X_{p t o} R_{i}}{R_{p t o}^{2}+X_{p t o}^{2}}
\end{array}\right.
$$

with $P_{a}^{* *} \rightarrow-\infty$, which is the global minimum, that is not of interest. 
Note that $P_{a}^{*}$ in (36) is a local maximum. Since the system of equations (34) has only two solutions, for which (37) is the global minimum, it follows that $P_{a}^{*}$ is the unique global maximum.

\subsection{Optimal Control - Robust Case}

In the uncertain WEC and PTO dynamics, the optimization problem becomes

$$
\max _{\left\{R_{c}, X_{c}\right\}} \min _{\left\{R_{i}, X_{i}, R_{p t o}, X_{p t o}\right\}} \frac{R_{p t o} R_{c}-X_{p t o} X_{c}}{\left(R_{i}+R_{p t o} R_{c}-X_{p t o} X_{c}\right)^{2}+\left(X_{i}+R_{p t o} X_{c}+X_{p t o} R_{c}\right)^{2}}
$$

where the factor $\frac{A^{2}}{2}$ is omitted, because it has no influence on the optimal solution. This is a difficult max-min optimization problem, since

- The cost function is not convex or concave everywhere.

- The parameters $R_{i}, X_{i}, R_{p t o}, X_{p t o}$ are not exactly known.

Before proceeding further, denote the values sets for $\left(R_{i}, X_{i}\right)$ and $\left(R_{p t o}, X_{p t o}\right)$ as

$$
\left\{\begin{array}{l}
\left(R_{i}, X_{i}\right) \in \quad\left\{\left\{R_{i}\right\},\left\{X_{i}\right\}\right\}, \\
\left(R_{p t o}, X_{p t o}\right) \in\left\{\left\{R_{p t o}\right\},\left\{X_{p t o}\right\}\right\}
\end{array}\right.
$$

We require that, for each given frequency $w$

$$
R_{p t o} R_{c}-X_{p t o} X_{c}>0, \forall R_{p t o}, \forall X_{p t o}
$$

since the harvested energy should be positive. Using (39), it is clear that (40) holds if and only if the following two conditions are satisfied

$$
\left\{R_{p t o}\right\} R_{c}-\left\{X_{p t o}\right\} X_{c}>0
$$

With the constraints (41), the cost function (38) is positive. It is hence possible to rewrite (38) as

$$
\min _{R_{c}, X_{c}} \max _{R_{i}, X_{i}, R_{p t o}, X_{p t o}} \frac{\left(R_{i}+R_{p t o} R_{c}-X_{p t o} X_{c}\right)^{2}+\left(X_{i}+R_{p t o} X_{c}+X_{p t o} R_{c}\right)^{2}}{R_{p t o} R_{c}-X_{p t o} X_{c}}
$$

where instead of solving a max-min optimization problem, we solve the equivalent min-max optimization problem with the inverse cost function.

The following theorem holds.

Theorem 2: The optimal solution to (42) can be obtained by solving the 
following convex SDP problem

$$
\begin{aligned}
& \min _{\gamma, R_{c}, X_{c}}\{\gamma\}, \\
& \text { s.t. }
\end{aligned}\left[\begin{array}{ccc}
\gamma & \left\{R_{i}\right\}+\left\{R_{p t o}\right\} R_{c}-\left\{X_{p t o}\right\} X_{c} & \left\{X_{i}\right\}+\left\{R_{p t o}\right\} X_{c}+\left\{X_{p t o}\right\} R_{c} \\
(*) & \left\{R_{p t o}\right\} R_{c}-\left\{X_{p t o}\right\} X_{c} & 0 \\
(*) & (*) & \left\{R_{p t o}\right\} R_{c}-\left\{X_{p t o}\right\} X_{c}
\end{array}\right] \succeq 0
$$

Proof: It is well known [2] that a quadratic over linear fractional function is convex. Hence (42) can be transformed into the following convex optimization problem

$$
\begin{aligned}
& \min _{\gamma, R_{c}, X_{c}}\{\gamma\}, \\
& \text { s.t. }\left\{\begin{array}{l}
\gamma \geq \frac{\left(R_{i}+R_{p t o} R_{c}-X_{p t o} X_{c}\right)^{2}+\left(X_{i}+R_{p t o} X_{c}+X_{p t o} R_{c}\right)^{2}}{R_{p t o} R_{c}-X_{p t o} X_{c}}, \\
R_{p t o} R_{c}-X_{p t o} X_{c}>0
\end{array}\right.
\end{aligned}
$$

where $\gamma$ is an auxiliary variable, that presents the upper bound of the cost function. Although convex, the optimization problem (44) is still difficult to solve due to the uncertain parameters $R_{i}, X_{i}, R_{p t o}, X_{p t o}$. Using Schur's complements, rewrite the constraints of (44) as

$$
\left[\begin{array}{ccc}
\gamma & R_{i}+R_{p t o} R_{c}-X_{p t o} X_{c} & X_{i}+R_{p t o} X_{c}+X_{p t o} R_{c} \\
(*) & R_{p t o} R_{c}-X_{p t o} X_{c} & 0 \\
(*) & (*) & R_{p t o} R_{c}-X_{p t o} X_{c}
\end{array}\right] \succeq 0
$$

Using (39), it is clear that (45) is satisfied if and only if the following conditions hold

$$
\left[\begin{array}{ccc}
\gamma & \left\{R_{i}\right\}+\left\{R_{p t o}\right\} R_{c}-\left\{X_{p t o}\right\} X_{c}\left\{X_{i}\right\}+\left\{R_{p t o}\right\} X_{c}+\left\{X_{p t o}\right\} R_{c} \\
(*) & \left\{R_{p t o}\right\} R_{c}-\left\{X_{p t o}\right\} X_{c} & 0 \\
(*) & (*) & \left\{R_{p t o}\right\} R_{c}-\left\{X_{p t o}\right\} X_{c}
\end{array}\right] \succeq 0
$$

Combining (41), (44), (46), it follows that the min-max problem (38) can be converted into the following SDP optimization problem

$$
\min _{\gamma, R_{c}, X_{c}}\{\gamma\}
$$

s.t. the constraints $(46)$

The proof is complete. 
In summary, in the presence of the uncertain WEC and PTO systems, the real part and the imaginary part of the optimal controller can be optimized using Algorithm 2.

$\overline{\text { Algorithm } 2 \text { : Calculation of the real part } R_{c}^{*} \text { and the imaginary part } X_{c}^{*} \text { of }}$ the robust optimal control block

1: Select a reasonable finite but large set of frequency samples $0<w_{1}<$ $\ldots<w_{N}$.

2: For each frequency in the chosen set

3: Compute the value sets $\left\{\left\{R_{i}\right\},\left\{X_{i}\right\}\right\}$ of the WEC intrinsic impedance.

4: Compute the value sets $\left\{\left\{R_{p t o}\right\},\left\{X_{p t o}\right\}\right\}$ of the PTO system.

5: $\quad$ Compute $R_{c}^{*}, X_{c}^{*}$ by solving the convex SDP problem (47).

Remark: The worst case optimal average power is computed as, for a given wave excitation force (19),

$$
P_{a}^{*}=\frac{A^{2}}{2 \gamma^{*}}
$$

where $\gamma^{*}$ is the optimal solution of $(47)$.

Fig. 4 presents the value sets $\left\{\left\{R_{p t o}\right\},\left\{X_{p t o}\right\}\right\}$ of a simple first-order uncertain PTO system (49) as a function of frequency. The PTO system is given as
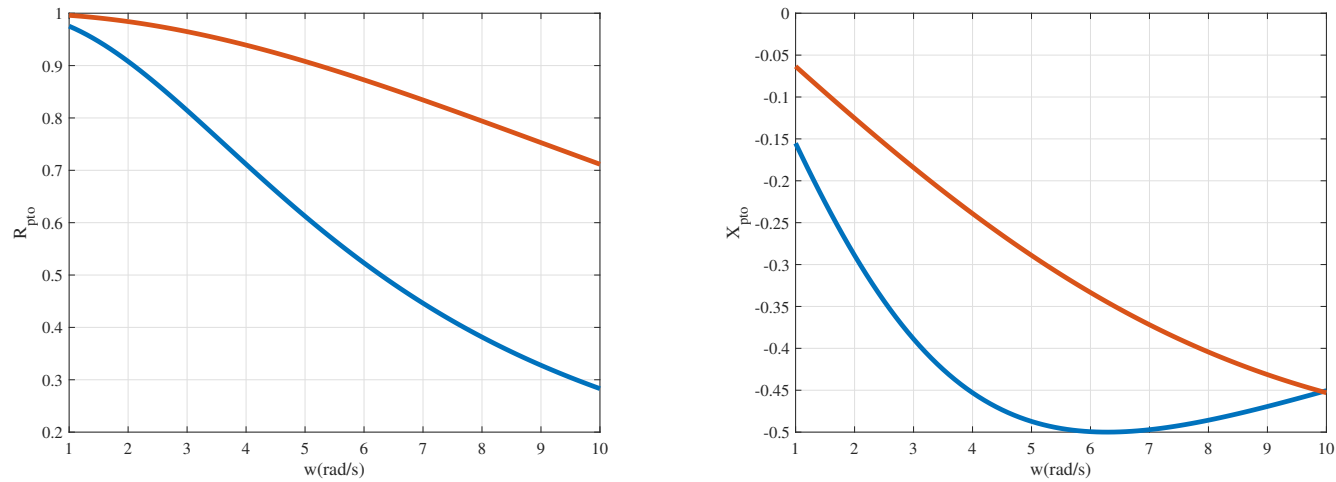

Fig. 4. Value sets $\left\{\left\{R_{p t o}\right\},\left\{X_{\text {pto }}\right\}\right\}$ of uncertain PTO system.

$$
W_{p t o}(j w)=\frac{1}{j w \tau+1}
$$

where $\tau$ is a uncertain time constant, $\tau \in\left[\frac{1}{5 \pi}, \frac{1}{2 \pi}\right]$. Note that in this case, the value sets $\left\{\left\{R_{p t o}\right\},\left\{X_{p t o}\right\}\right\}$ can be computed analytically as function of frequency $w$.

Using CVX to solve the SDP optimization problem (47), one obtains the value $R_{c}^{*}, X_{c}^{*}$ of the optimal controller, see Fig. 5. These two figures also present the value sets of $R_{i}$ and of $-X_{i}$ of the WEC system. The parameters of the WEC system are given in the simulation section. 


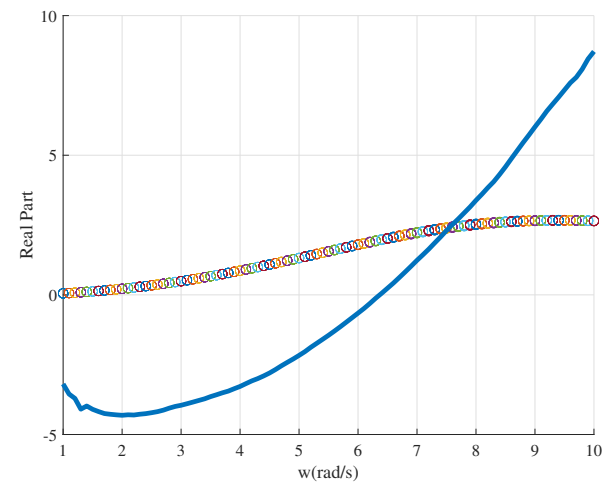

(a) Optimal $R_{c}^{*}$ and $R_{i}$

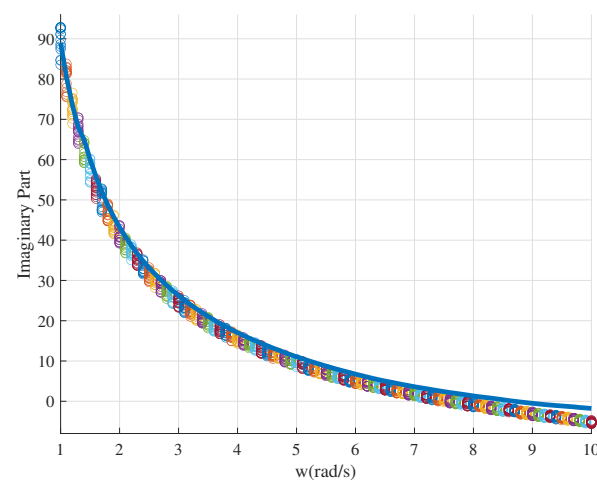

(b) Optimal $X_{c}^{*}$ and $-X_{i}$

Fig. 5. (a) Optimal $R_{c}^{*}$ (solid blue) and $R_{i}$ (circle marker), (b) $X_{c}^{*}$ (solid blue) and $-X_{i}(($ circle marker $))$ as a function of $\omega$.

Remark: It is well known that [5], in the nominal case and without the PTO dynamics, the optimal WEC velocity is is phase with the wave excitation force. Clearly, in the robust case, there is no guarantee that there exists a control law (20) such that the imaginary part of the transfer function $W(j w)$ is zero. It implies that the velocity is generally not in phase with the wave excitation force in the robust case.

\section{Extension to Polychromatic Waves}

We show how to apply the results in Section 3 to polychromatic sea states in this section. For this purpose, the following three main problems need to be addressed.

(1) The wave excitation force $f_{e x}(t)$ is not directly accessible when the WEC system is running.

(2) Real sea states are polychromatic, i.e., $f_{e x}(t)$ is not a pure sine.

(3) A control structure needs to be assigned for $Z_{c}(j w)$.

For the first problem, a few methods have been proposed and tested in realtime to estimate the wave excitation force via measurements of other quantities [12], [1], [20]. In this work, a method in [20] is used. It is based on a linear Kalman filter and a random walk model for the variation of the wave excitation force. Only the float position, the float velocity, and the PTO force are required. The method yields accurate estimates over a large range of sea states.

The second problem is resolved by approximating $f_{e x}(t)$ as a sinusoidal signal 
with time-varying amplitude $A(t)$, angular frequency $w(t)$ and phase $\phi(t)$, i.e.

$$
f_{e x}(t)=A(t) \sin (w(t) t+\phi(t))
$$

The parameters $A(t), w(t)$ and $\phi(t)$ are estimated on-line. It is clear that this is a nonlinear estimation problem, since $A(t), w(t)$ and $\phi(t)$ enter nonlinearly in $(50)$.

It should be stressed that the problem of estimating the unknown parameters describing a noisy sinusoidal signal is an important one in systems theory with applications in diverse fields [26]. The most of the existing solutions can be listed here are: line enhancers [30], finite impulse response filters [32], infinite impulse response filters or notch filters [27], and frequency locked loop [11].

In this paper an unscented Kalman filter (UKF) [24] is used to estimate $A(t), w(t), \phi(t)$. The main advantages of the UKF filter are: i) the computational complexity is very small, hence it can be implemented in real-time, ii) it requires a modest memory footprint, since only $f_{e x}(t)$ at current time is needed, iii) it is already evaluated experimentally on real-data [23] with a good performance. Note that, like the extended Kalman filter, the UKF consists of the same two steps: state prediction and measurement correction, except they are preceded now by another step for the calculation of sigma points.

For the third problem, the following proportional integral (PI) control structure is chosen

$$
Z_{c}(j w)=K_{p}+\frac{K_{i}}{j w}
$$

Hence, in the frequency domain

$$
F_{u}(j w)=Z_{c}(j w) V(j w)=\left(K_{p}+\frac{K_{i}}{j w}\right) V(j w)
$$

or equivalently, in the time domain

$$
f_{u}(t)=K_{p} v(t)+K_{i} \int_{0}^{t} v(\tau) d \tau
$$

Hence

$$
f_{u}(t)=K_{p} v(t)+K_{i} p(t)
$$

where $p(t)=\int_{0}^{t} v(\tau) d \tau$ is the float position, which is assumed to be available in real time.

Combining (21) and (51), one obtains

$$
\left\{\begin{array}{l}
K_{p}=R_{c}^{*}, \\
K_{i}=-w X_{c}^{*}
\end{array}\right.
$$


The robust optimal PI control strategy is summarized into two stages: offline stage and online stage.

\section{Offline Stage}

1: $\quad$ Using Algorithm 2 to calculate the optimal $R_{c}^{*}, X_{c}^{*}$.

2: $\quad$ Calculate the optimal gains $K_{p}, K_{i}$ as in (54).

\begin{tabular}{ll}
\hline Online Stage: At each sampling instant $t$ \\
\hline $1:$ & Estimate $f_{e x}(t)$ using $v(t), p(t)$ and $f_{p t o}(t)$. \\
$2:$ & Estimate the frequency $\hat{\omega}$ using the UKF. \\
$3:$ & The optimal gains $K_{p}, K_{i}$ are found by a look-up table from \\
& the offline stage. \\
$4:$ & The control action is computed as: $f_{u}(t)=K_{p} v(t)+K_{i} p(t)$ \\
\hline
\end{tabular}

The robust adaptive PI control algorithm is schematically presented in Fig. 6 . It is worth noticing that the algorithm requires only information about $p(t), v(t)$ and $f_{p t o}(t)$. No information about $f_{e x}(t)$ is needed.

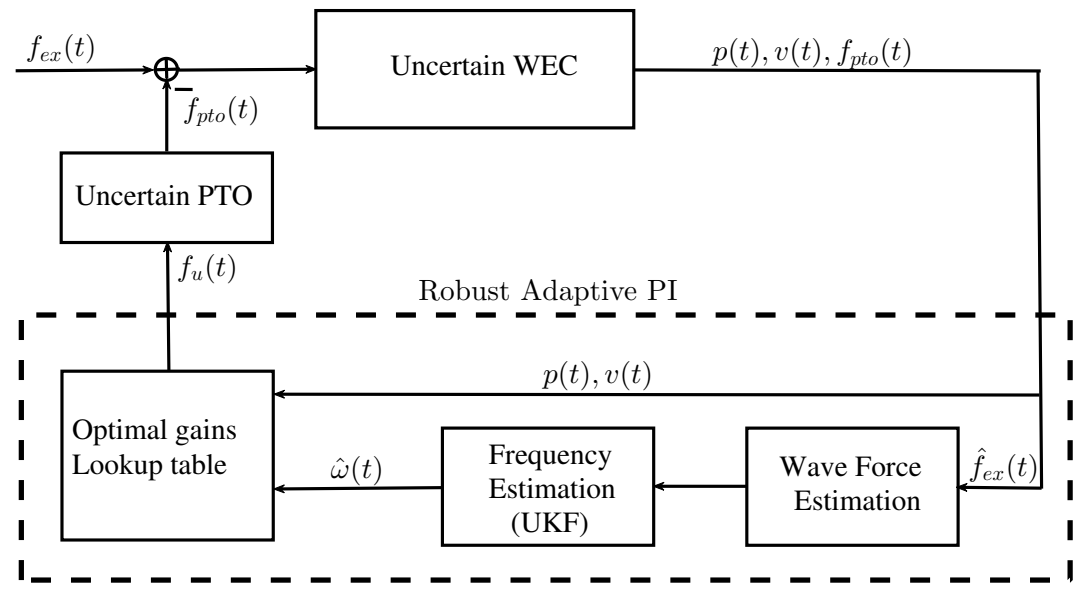

Fig. 6. Robust adaptive PI control structure.

Remark: For simplicity and for a clear physical interpretation, the PI control structure is used in this work. As written in the introduction, the wave energy is converted into useful energy with the $\mathrm{P}$-component, why the I-component modifies the WEC system natural frequency. Other high order control structure such as PID can also be used. The main advantage of these high order control laws is that they offer an additional degree of freedom to harvest more energy. 


\section{Simulation Results}

\subsection{WEC Parameters}

A laboratory prototype of a point absorber WEC [33] on a 1:20 scale with respect to the well-known Wavestar machine installed near Hanstholm in Denmark from 2009 to 2013, was used to validate the robust adaptive PI control algorithm described in Section 4. The WEC parameters are given as $M=1.44$, $K_{v}=0.01,85 \leq K_{h d} \leq 95$, and

$$
W_{r}(j w)=\frac{a_{4}(j w)^{4}+a_{3}(j w)^{3}+a_{2}(j w)^{2}+a_{1}(j w)+a_{0}}{b_{5}(j w)^{5}+b_{4}(j w)^{4}+b_{3}(j w)^{3}+b_{2}(j w)^{2}+b_{1}(j w)+b_{0}}
$$

whose coefficients are given in Table 5.1.

\begin{tabular}{|l|l|}
\hline Numerator & Denominator \\
\hline & $b_{5}=1$ \\
$a_{4}=30.8619$ & $b_{4}=208.5959$ \\
$a_{3}=6.4642 \times 10^{3}$ & $b_{3}=8.5834 \times 10^{4}$ \\
$a_{2}=2.6016 \times 10^{6}$ & $b_{2}=8.8994 \times 10^{6}$ \\
$a_{1}=2.6550 \times 10^{8}$ & $b_{1}=1.0740 \times 10^{8}$ \\
$a_{0}=-7.0967 \times 10^{5}$ & $b_{0}=7.0311 \times 10^{8}$ \\
\hline
\end{tabular}

Using Algorithm 1, Fig. 7 presents the Bode plot of the considered WEC system. Note that this Bode plot is very typical for a WEC system of the point-absorber type.

\subsection{Simulation Results}

In this section, we present the simulation results obtained using the robust adaptive control algorithm descried in Section 4. To validate our concept, we choose one sea state, that represents a normal operating condition for the WaveStar WEC. Fig. 8 shows the spectral power density of this wave.

Fig. 9 presents the estimation results of the UFK filter. Recall that the UKF filter is used to estimate the wave frequency as well as the wave amplitude. It can be observed that a very satisfactory performance of the UKF filter is obtained. 

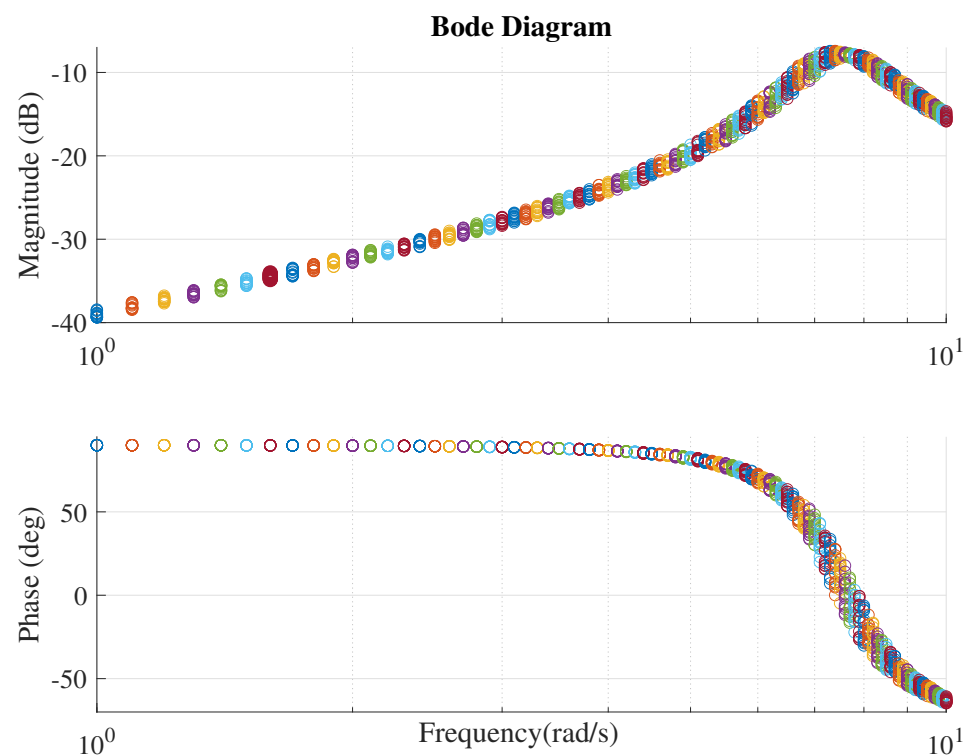

Fig. 7. Bode plot of the considered WEC system.

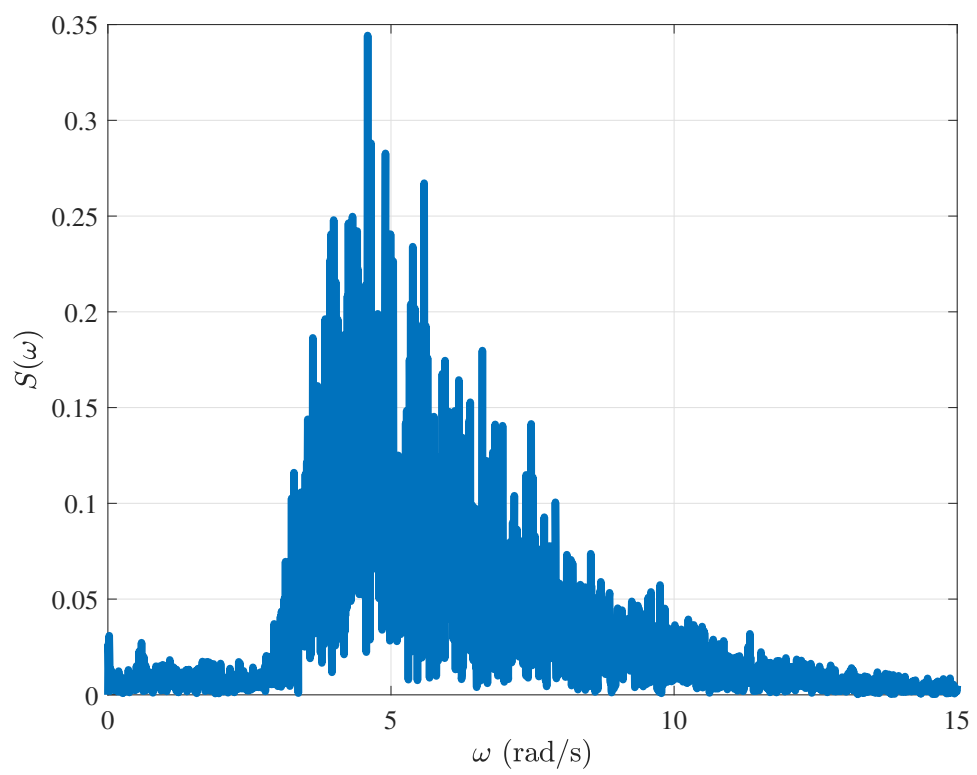

Fig. 8. Wave spectral power density.

The transfer function of the PTO system with a uncertain time constant is given in (49).

For 20 different realizations of $K_{h d}$ and $\tau$, see Fig. 10, Fig. 11 shows, respectively, different WEC positions and WEC velocities as a function of time using the robust adaptive control law.

Fig. 12(a) presents the harvested energy obtained as the integral of the in- 


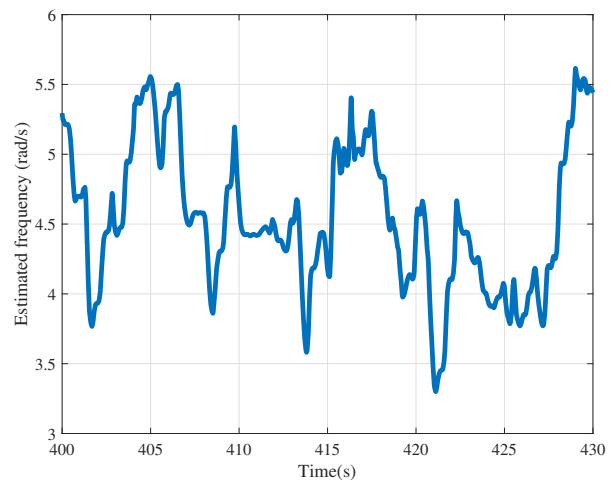

(a)Frequency

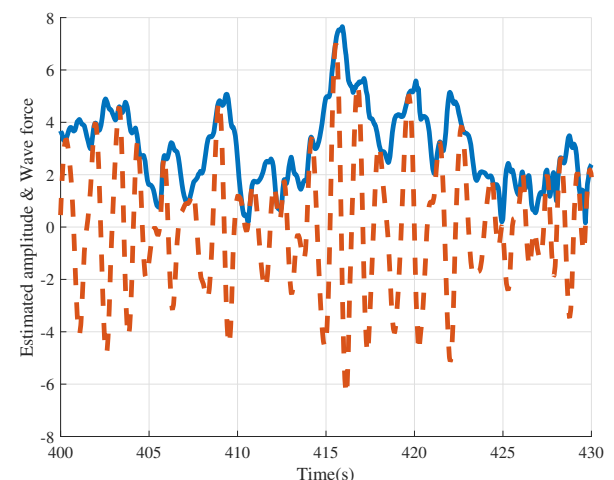

(b)Amplitude \& Wave force

Fig. 9. (a) Estimated frequency, (b) Estimated amplitude (solid blue) and wave excitation force (dashed red).

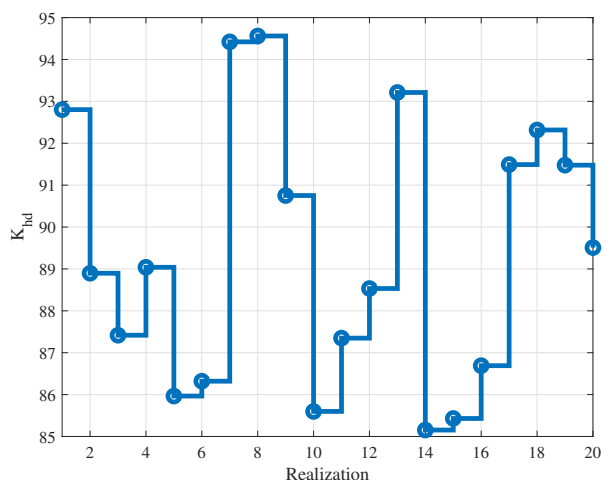

(a) $K_{h d}$

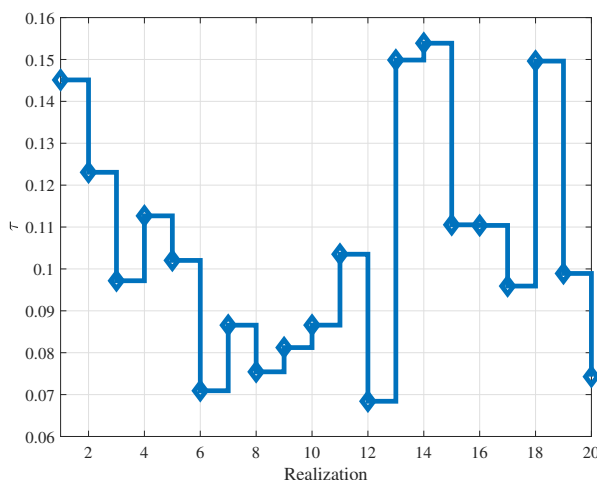

(b) $\tau$

Fig. 10. 20 different realizations of $K_{h d}$ and $\tau$.

stantaneous power. For comparison, Fig. 12(b) shows the harvested energy obtained applying the nominal adaptive PI control law in Section 3.2. Using $12(\mathrm{~b})$, it is clear that if uncertainties are not properly taken into account in the controller design step, the harvested energy might be negative. For the nominal adaptive controller, $R_{i}$ and $X_{i}$ are chosen as the mean of $\left\{R_{i}\right\}$, and of $\left\{X_{i}\right\}$, respectively. The nominal PTO system is selected as

$$
W_{p t o}(j w)=\frac{1}{3.5(j w)+1}
$$

\section{Conclusion}

A new control approach to the problem of maximizing the harvested energy with a point absorber in the presence of a uncertain WEC and PTO dynamics 


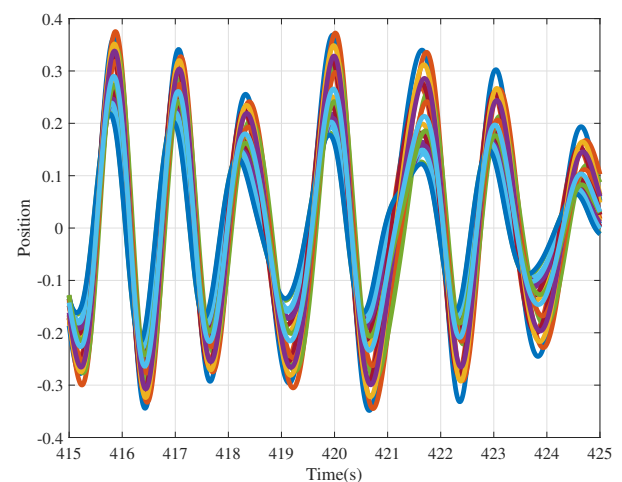

(a)Position

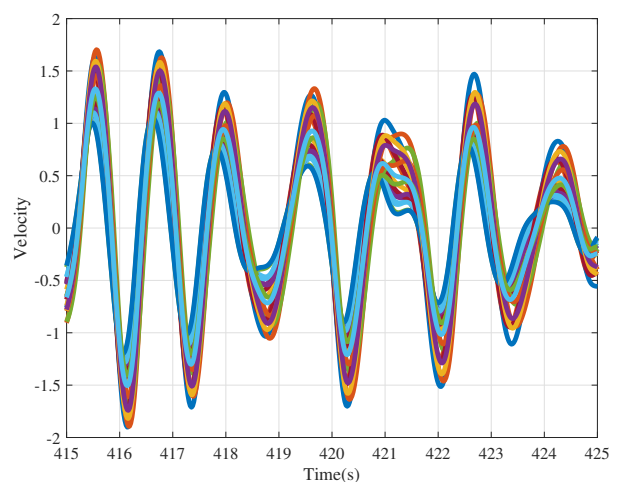

(b)Velocity

Fig. 11. Buoy position and velocity for 20 different realizations of $K_{h d}$ and $\tau$.

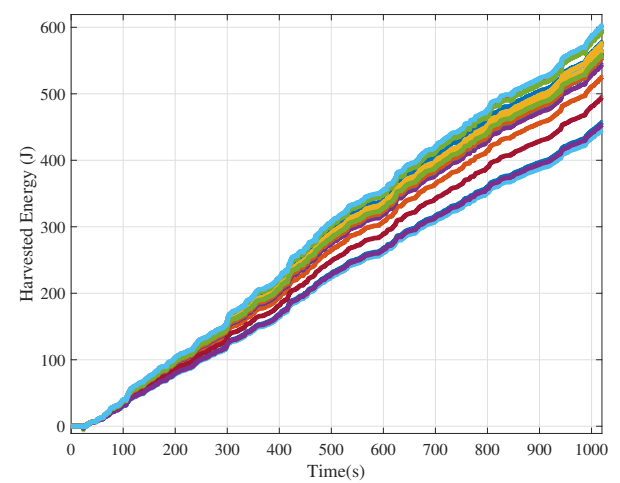

(a)Robust Adaptive

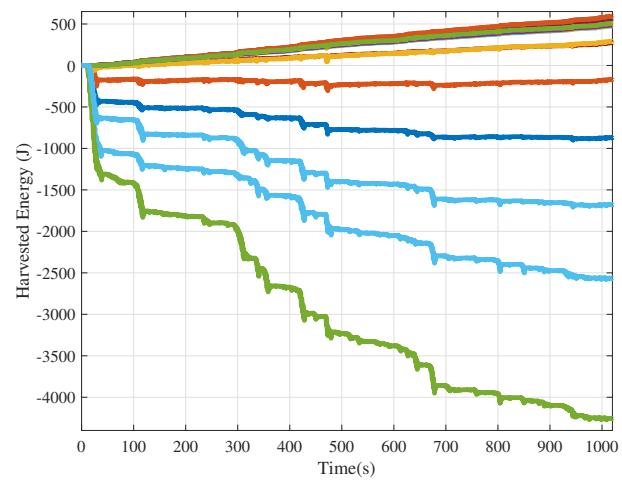

(b)Nominal Adaptive

Fig. 12. Harvested energy for 20 different realizations of $K_{h d}$ and $\tau$.

is proposed. The basic idea is to adopt a robust adaptive PI control strategy. The design procedure is decomposed into an offline and online stages. In the offline stage, the optimal PI gains are computed by maximizing the worst-case harvested average power. It is shown that this problem can be converted into a convex semidefinite optimization problem, for which the optimal solution is unique. In the online stage, by approximating the wave excitation force as a time-varying sinusoid, the gains of the PI controller are continuously adapted on-line, on a wave-to-wave basis. A simulation example is given and compared to earlier solutions from literature.

We will in the future, consider the input and output constraints. We are also interested in extending this technique to the multi-float configuration case. 


\section{References}

[1] O Abdelkhalik, S Zou, R Robinett, G Bacelli, and D Wilson. Estimation of excitation forces for wave energy converters control using pressure measurements. International Journal of Control, 90(8):1793-1805, 2017.

[2] Stephen Boyd and Lieven Vandenberghe. Convex optimization. Cambridge university press, 2004.

[3] WE Cummins. The impulse response function and ship motions. Technical report, DTIC Document, 1962.

[4] Benjamin Drew, AR Plummer, and M Necip Sahinkaya. A review of wave energy converter technology. Proceedings of the Institution of Mechanical Engineers, Part A: Journal of Power and Energy, 223(8):887-902, 2009.

[5] Johannes Falnes. Ocean waves and oscillating systems: linear interactions including wave-energy extraction. Cambridge university press, 2002.

[6] Francesco Fusco and John V Ringwood. Hierarchical robust control of oscillating wave energy converters with uncertain dynamics. IEEE Transactions on Sustainable Energy, 5(3):958-966, 2014.

[7] Noam Galperin, Per-Olof Gutman, and Hector Rotstein. Value set identification using lissajou figure sets. IFAC Proceedings Volumes, 29(1):4016-4021, 1996.

[8] Michael Grant and Stephen Boyd. Cvx: Matlab software for disciplined convex programming.

[9] Howard Gruenspecht. International energy outlook 2011. Center for Strategic and International Studies, 2010.

[10] Jørgen Hals, Johannes Falnes, and Torgeir Moan. A comparison of selected strategies for adaptive control of wave energy converters. Journal of Offshore Mechanics and Arctic Engineering, 133(3):031101, 2011.

[11] Guan-Chyun Hsieh and James C Hung. Phase-locked loop techniques. a survey. IEEE Transactions on industrial electronics, 43(6):609-615, 1996.

[12] Peter Kracht, Sebastian Perez-Becker, Jean-Baptiste Richard, and Boris Fischer. Performance improvement of a point absorber wave energy converter by application of an observer-based control: Results from wave tank testing. IEEE Transactions on Industry Applications, 51(4):3426-3434, 2015.

[13] Chang-Ho Lee and J Nicholas Newman. Wamit user manual. WAMIT, Inc, 2006.

[14] Lennart Ljung. System Identification: Theory for the User. Pearson Education, 1998.

[15] Johan Lofberg. Yalmip: A toolbox for modeling and optimization in matlab. In Computer Aided Control Systems Design, 2004 IEEE International Symposium on, pages 284-289. IEEE, 2004. 
[16] Ardashir Mohammadzadeh and Tufan Kumbasar. A new fractional-order general type-2 fuzzy predictive control system and its application for glucose level regulation. Applied Soft Computing, 91:106241, 2020.

[17] Ardashir Mohammadzadeh and Sakthivel Rathinasamy. Energy management in photovoltaic battery hybrid systems: A novel type-2 fuzzy control. International Journal of Hydrogen Energy, 45(41):20970-20982, 2020.

[18] Annette Muetze and JG Vining. Ocean wave energy conversion-a survey. In Industry Applications Conference, 2006. 41st IAS Annual Meeting. Conference Record of the 2006 IEEE, volume 3, pages 1410-1417. IEEE, 2006.

[19] H-N Nguyen and P Tona. Robust adaptive pi control of wave energy converters with uncertain pto systems. In 2018 IEEE Conference on Decision and Control $(C D C)$, pages 5518-5523. IEEE, 2018.

[20] H-N Nguyen and Paolino Tona. Wave excitation force estimation for wave energy converters of the point-absorber type. IEEE Transactions on Control Systems Technology, 2017.

[21] Hoai-Nam Nguyen. Constrained Control of Uncertain, Time-Varying, DiscreteTime Systems: An Interpolation-Based Approach, volume 451. Springer, 2013.

[22] Hoai-Nam Nguyen and Paolino Tona. Continuously adaptive PI control of wave energy converters under irregular sea-state conditions. In 12th European Wave and Tidal Energy Conference 2017, 2017.

[23] Hoai-Nam Nguyen and Paolino Tona. An efficiency-aware continuous adaptive proportional-integral velocity-feedback control for wave energy converters. Renewable Energy, 2019.

[24] Hoai-Nam Nguyen, Paolino Tona, and Guillaume Sabiron. Dominant wave frequency and amplitude estimation for adaptive control of wave energy converters. In OCEANS 2017-Aberdeen, pages 1-6. IEEE, 2017.

[25] Arthur Pecher and Jens Peter Kofoed. Handbook of Ocean Wave Energy. Springer, 2017.

[26] Barry G Quinn and Edward James Hannan. The estimation and tracking of frequency. Number 9. Cambridge University Press, 2001.

[27] Phillip A Regalia. An improved lattice-based adaptive iir notch filter. IEEE transactions on signal processing, 39(9):2124-2128, 1991.

[28] Enrique Vidal Sánchez, Rico Hjerm Hansen, and Morten Mejlhede Kramer. Control performance assessment and design of optimal control to harvest ocean energy. IEEE Journal of Oceanic Engineering, 40(1):15-26, 2015.

[29] Torsten Söderström and Petre Stoica. System identification. Prentice-Hall, Inc., 1988.

[30] J Treichler. Transient and convergent behavior of the adaptive line enhancer. IEEE Transactions on Acoustics, Speech, and Signal Processing, 27(1):53-62, 1979. 
[31] A Wahyudie, MA Jama, O Saeed, H Noura, A Assi, and K Harib. Robust and low computational cost controller for improving captured power in heaving wave energy converters. Renewable Energy, 82:114-124, 2015.

[32] Bernard Widrow, John R Glover, John M McCool, John Kaunitz, Charles S Williams, Robert H Hearn, James R Zeidler, JR Eugene Dong, and Robert C Goodlin. Adaptive noise cancelling: Principles and applications. Proceedings of the IEEE, 63(12):1692-1716, 1975.

[33] Andrew S Zurkinden, Morten Kramer, Mahdi Teimouri Teimouri, and Marco Alves. Comparison between numerical modeling and experimental testing of a point absorber wec using linear power take-off system. In ASME 201231 st International Conference on Ocean, Offshore and Arctic Engineering, pages 497-506. American Society of Mechanical Engineers, 2012. 